It is interesting in the same connexion to note a report by Howard, Frazier and Miles (J. Biol. Chem., 241,4293 ; 1966) of the formation of a two-stranded helix between poly-U and poly-2-aminoadenylic acid. The latter is merely poly-A containing an amino-group in a position such that a good hydrogen bond can be made with the 02 of the uracil without significantly distorting the two usual hydrogen bonds between adenine and uracil. It may be an oversimplification to suggest that the effect on the stability of the helix arises only from the energy of the new hydrogen bond, with no contribution, for example, from the change in dipole moment. Nevertheless, a stabilization amounting to a $25^{\circ}$ increase in melting temperature is observed. A recent article by Nash and Bradley (J. Chem Phys., 45, 1380; 1966) starts from the view that the coulombic effect on the stability of base pair configuration is dominant. Semi-empirical molecular orbital procedures are used to calculate point charges on the atoms of the four bases (A, U, Gr and $\mathrm{C}$ ), and the coulombic interaction potentials generated for all possible pairs as one base is rotated relative to its partner are then computed. The calculation produces a total of twenty-seven deep potential wells, and the authors are gratified to note that in general these correspond to highly linear $\mathrm{N}-\mathrm{H}$. . O and $\mathrm{N}-\mathrm{H}$. . N contacts, so that hydrogen bonds are again energetically advantageous in the coulombically favoured geometries. Whatever the relative strengths of the hydrogen bonds between base and base and between base and water, it is in any case clear that if good hydrogen bonds could not in fact be made in the double-helix, the helical state would be much less favoured relative to the coil.

\section{Meeting about Meteorites}

In the early years of the United States space programme it sometimes seemed as if the study of meteorites was almost entirely meant to understand the nature of the Moon's surface and to help solve re-entry problems. The subjects discussed at the Twenty-ninth Annual Meeting of the Meteoritical Society, held in Washington, D.C., during November 3-5, 1966, however, were notable for their academic liveliness. The only paper not concerned with the study of meteorites for their own sakes dealt with the discovery that ceremonial Chinese weapons of the tenth to eighth centuries B.c. were made of meteoritic iron and did not indicate a flourishing iron-forging industry.

The conference produced additional evidence on the now familiar problem of tektites, but unfortunately the new evidence supported both sides of the argument equally. Tektites are glassy disks or Jumps which are found spread over wide areas or "fields", principally in Central Europe, the Far East and Australasia. Although chemically similar to one another, they do not resemble any one terrestrial rock in all facets of their chemistry. There is general agreement that they were produced by fractional melting (with loss of volatiles), probably as a result of meteoritic impact. The current controversy is whether this melting on impact occurred at the surface of the Moon or the Earth.

The meeting moved from the serious consideration of tektites to the serious consideration of two ideas regarded as "cranky" by more conservative geologists. One idea is that carbonaceous chondrites may come from comets, not asteroids. The other concerns the formation of the Sudbury Basin (Ontario), for which a mechanism of meteoritic impact has recently been suggested. Such a mechanism implies that petrographic features induced by shock can be preserved for $1,500 \times$ $10^{6} \mathrm{yr}$; that shatter cones are a positive criterion of meteoritic impact; and that meteoritic impact may produce or trigger igneous activity which involves large volumes of magma and ores.

The conference was also told of a freak discovery. Pieces of native copper, thought to represent fragments of a meteorite weighing at least $108 \mathrm{lb}$., have come to light in British Columbia. By any standards this meteorite is a rare find-so long as it is genuinely extraterrestrial.

At the meeting authors were given 10 minutes to read their papers, and this was to be followed by a 5 minute period for discussion. Four authors out of five, however, overran their time, thus leaving little or no time for discussion. That many discussion periods were cut or abandoned altogether is all the sadder because (as one delegate put it) "informal discussions are almost the real justification for meetings like this".

\section{Pure Cultures}

A CONFERENCE under the label of "Axenic Cultures and Defined Media", held by the New York Academy of Sciences (Ann. N.Y. Acad. Sci., 139, 1; 1966), has usefully co-ordinated some of the information available. about techniques for the culture of cells, tissues and organisms. Axenic cultures are ones free from contamination by species other than those of interest and are of considerable social and economic importance. Contributions to the conference were mainly concerned with progress in the perfection of the techniques involved, but the feasibility of their application to such problems as the development of antibiotics and insecticides, and vaccines and toxins against parasites, was also stressed.

The culture of parasites, both in infected tissue and free from their hosts, is vital for the investigation of life histories and behaviour. Avian embryos and various animal tissues are now used for the axenic culture of many protozoan parasites such as Plasmodium and Trypanosoma (responsible for malaria and sleeping sickness, respectively). The aim is to produce from these cultures antigens to be used for immunization against infection by the parasites. Multi-cellular parasites are also of interest in this respect; the immunity of vertebrate animals to infection by hel. minths (flat worms) comes mainly from antigens produced at the transition from third to fourth and fourth to fifth free living larval stages. Culture techniques for these larvae, which involve specific media and controlled gas atmospheres, are being developed to facilitate economic production of the antigens.

The purity of axenic cultures of parasites make them ideal for treatment with chemicals which may be notential toxins. Toxic effects are obviously caused by the added compound and not by bacterial, viral or other activity resulting from infection. The plant nematode (round worm) Pratylenchus penetrans can be grown on excised plant roots, and an apparatus to provide the necessary micro-environment for the maintenance of viable populations of the parasite is in development. When the nematodes are freely available in an uncontaminated condition they can be used for 